\title{
Flow Cytometry Approach for the Identification of Liposomal Cytarabine Toxicity
}

\author{
Dolores Subiráa ${ }^{a}$, Isabel López San Romána, Dunia de Miguela ${ }^{a}$ Miguel Díaz-Morfa ${ }^{a}$, \\ Isabel Izquierdo ${ }^{\mathrm{b}}$, Nuria Golbano ${ }^{\mathrm{a}}$, Carolina Cerrato ${ }^{\mathrm{a}}$, M. Dolores Morales ${ }^{\mathrm{a}}$, \\ Sonia Herrero ${ }^{a}$, Jaime Arbeteta ${ }^{\mathrm{a}}$, Felix Fuertes ${ }^{\mathrm{a}}$, Blanca Pinedo ${ }^{\mathrm{a}}$
}

\begin{abstract}
Neurological complications may develop either at diagnosis or during the course of many hematological malignancies after initiation of therapy. The etiology might be very different but, once infection is excluded, differential diagnosis is mainly focused on distinguishing between leptomeningeal progression and toxicity secondary to therapy. In each case, the subsequent clinical decision would be completely opposite. Contrast central nervous system magnetic resonance, biochemistry, serology and cultures of the cerebrospinal fluid, and electromyography are usually performed in these patients, but demonstration of toxicity remains a diagnosis of exclusion for reagents such as liposomal cytarabine. Based on the experience of 2 clinical cases, we reviewed a database of immunophenotypic studies of the cerebrospinal fluid, and then we summarize how to use flow cytometry to positively support liposomal cytarabine toxicity.
\end{abstract}

Keywords: Flow cytometry; Cerebrospinal fluid; Liposomal cytarabine toxicity

\section{Introduction}

Patients with haematological malignancies may develop neurological complications either at diagnosis or during the

Manuscript accepted for publication April 6, 2012

\footnotetext{
${ }^{a}$ Department of Hematology, Hospital de Guadalajara, c/Donantes de sangre s/n. Guadalajara 19002, Spain

${ }^{b}$ Department of Hematology, Hospital Santa Creu i Sant Pau, Sant Antoni Maria Claret, 167. 08025 Barcelona, Spain

${ }^{\mathrm{c}}$ Corresponding author: Dolores Subirá, Department of Haematology,

Flow Cytometry Division, Hospital Universitario de Guadalajara,

c/ Donantes de sangre s.n. Guadalajara 19002, Spain.

Email: dosuperez@yahoo.es
}

doi:10.4021/jnr95w course of their disease $[1,2]$. Current diagnostic methods include biochemical analysis, serology and cultures for identification of metabolic abnormalities and infectious diseases, contrast central nervous system (CNS) magnetic resonance imaging (MRI), and cerebrospinal fluid (CSF) studies for detecting cerebrovascular diseases and CNS metastases or leptomeningeal dissemination. Electromyography will help to identify paraneoplastic syndrome, and measurement of the levels of some drugs used in therapy protocols (i.e. methotrexate) can be used to discard toxicity. However, toxicity due to other agents, such as liposomal cytarabine (LC, Depocyte ${ }^{\circledR}$ ) remains a diagnosis of exclusion and a clinical dilemma.

Flow cytometry (FC) immunophenotyping is a very useful tool for the examination of the CSF [3-7]. It is generally used for the identification and characterization of cells, but it is also sensitive enough to detect other CSF particles. We present two patients in whom clinical suspicion of LC toxicity was supported by the FC findings in their CSF samples. A retrospective analysis of $\mathrm{FC}$ files from a database of CSF samples helped to establish the specificity of the findings obtained in our patients. We believe that recognition of this image could help to distinguish CNS involvement by a hematologic malignancy from clinical symptoms secondary to LC toxicity.

\section{Patients and Methods}

\section{Case 1}

A 46-year-old woman was diagnosed of IgA-multiple myeloma with a complex karyotype, stage IIIB. She received 5 courses of bortezomib (BZ)/cyclophosphamide (CF)/ dexamethasone (DX) and achieved a complete response: negative immunofixation and FC residual disease $<0.01 \%$. One week after peripheral stem cell apheresis, she presented with progressive peripheral neuropathy (left peripheral facial palsy, paresia and hyposthesia of the upper left limb plus dysphagia), and subcutaneous nodules that corresponded to plasmocytomas, as shown histologically. FC immunophenotyping ruled out bone marrow (BM) relapse, but revealed a 
Table 1. Patients' Clinical Symptoms and CSF Data Obtained After the Administration of the LC Doses

\begin{tabular}{|c|c|c|}
\hline & Patient 1 & Patient 2 \\
\hline Sex & $\mathrm{F}$ & M \\
\hline Age & 46 & 63 \\
\hline Diagnosis & Multiple Myeloma & $\begin{array}{l}\text { Mixed phenotype acute } \\
\text { leukemia (B/myeloid) }\end{array}$ \\
\hline CNS involvement at diagnosis & No & No \\
\hline Radiotherapy & No & No \\
\hline Neurotoxic drugs received & MTX, high dose Ara-C & MTX \\
\hline Diagnosis of leptomeningeal relapse & CSF cytology and FC & MRI \\
\hline \multicolumn{3}{|c|}{ Monitoring leptomeningeal disease } \\
\hline IT LC doses received & 7 & 5 \\
\hline Neurotoxicity & No & No \\
\hline CSF cell count & $<1 / \mathrm{mm}^{3}$ & $<1 / \mathrm{mm}^{3}$ \\
\hline Estimated ratio FC events: cells/depo foam & $38 / 79$ & $18 / 1,110$ \\
\hline IT LC doses received & 8 & 6 \\
\hline Neurotoxicity & No & Difficulty in walking \\
\hline CSF cell count & $2 / \mathrm{mm}^{3}$ & $<1 / \mathrm{mm}^{3}$ \\
\hline Estimated ratio FC events: cells/depo foam & $201 / 774$ & $12 / 1,013$ \\
\hline IT LC doses received & 10 & 7 \\
\hline Neurotoxicity & Cauda equina syndrome & Sacral radiculopathy \\
\hline CSF cell count & $1 / \mathrm{mm}^{3}$ & $1 / \mathrm{mm}^{3}$ \\
\hline Estimated ratio FC events: cells/depo foam & $17 / 13,888$ & $112 / 6,890$ \\
\hline CNS relapse & No & No \\
\hline Follow-up (months after neurological episode) & 4 & 13 \\
\hline Hematological status & $\begin{array}{l}\text { Extra-medullary relapse. Exitus } \\
\text { during allogeneic transplantation }\end{array}$ & Complete remission \\
\hline Neurological sequel & - & $\begin{array}{l}\text { Faecal and urinary } \\
\text { incontinence. }\end{array}$ \\
\hline
\end{tabular}

F: female; M: male; IT: intrathecal; LC: liposomal cytarabine; CNS: central nervous system; MTX: methotrexate; CSF: cerebrospinal fluid; FC: flow cytometry; MRI: magnetic resonance imaging. 
A

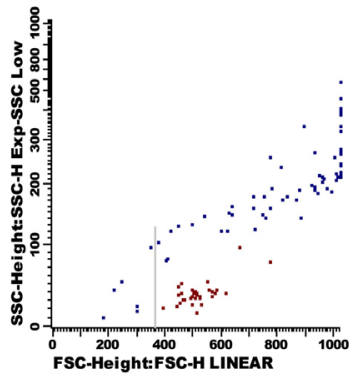

Cell-count: $<1 / \mathrm{mm} 3$ $\mathrm{N}^{\circ}$ doses cytarabine liposomal: 7

D

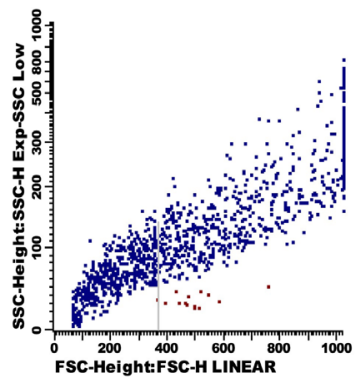

Cell-count: $<1 / \mathrm{mm} 3$

$\mathrm{N}^{\circ}$ doses cytarabine liposomal: 5
B

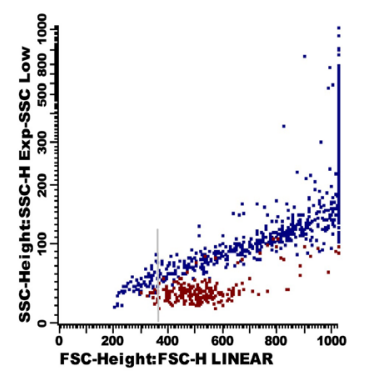

Cell-count: $2 / \mathrm{mm} 3$

$\mathrm{N}^{\circ}$ doses cytarabine liposomal: 8

E

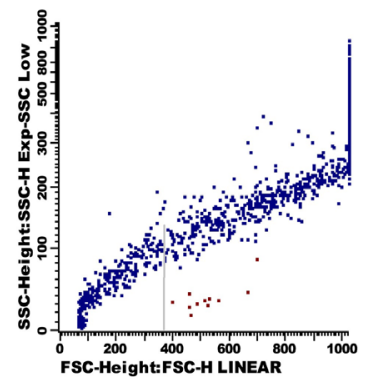

Cell-count: $<1 / \mathrm{mm} 3$

№ doses cytarabine liposomal: 6
C

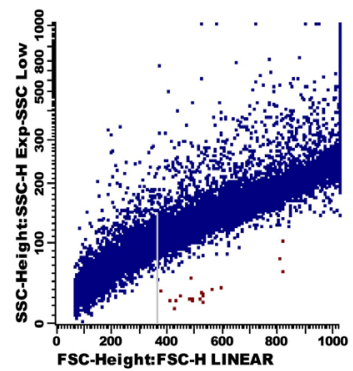

Cell-count: $1 / \mathrm{mm} 3$

$\mathrm{N}^{\circ}$ doses cytarabine liposomal: 10

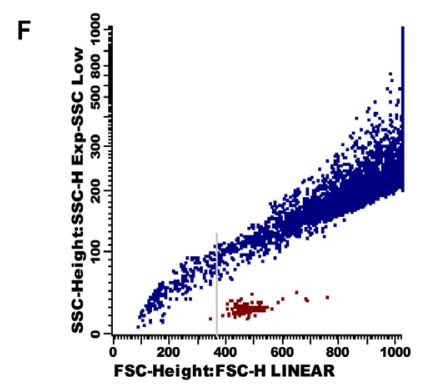

Cell-count: $1 / \mathrm{mm} 3$

$\mathrm{N}^{\circ}$ doses cytarabine liposomal: 7

Figure 1. Forward and side scatter dot-plots. Cells are coloured in red and liposomes in blue. These last events show a very wide range of size and granularity, and cross the forward/side scatter dot-plot from very small to very high and complex events. The grey arrow indicates the cut-off point for quantification of the depo-foam particles. Dot-plots A), B) C) belong to Patient 1, and dot-plots D), E) F) belong to Patient 2.

raised cell-count in the CSF with $20 \%$ of aberrant myeloma cells. A diagnosis of leptomeningeal myelomatosis and extra-medullary relapse was established, and she was started on systemic chemotherapy with topotecan $\left(1.5 \mathrm{mg} / \mathrm{m}^{2}\right.$, days $1,5)$, vincristine $\left(1.4 \mathrm{mg} / \mathrm{m}^{2}\right.$, day 1$)$, methotrexate (MTX, 8.4 $\mathrm{g} / \mathrm{m}^{2}$, day 2$)$, DX ( $8 \mathrm{mg} / 6$ hours, days 1 - 5) and thalidomide $(100 \mathrm{mg} /$ day). She also received 8 doses of intrathecal LC), (50 mg x 4/14 days, and $50 \mathrm{mg} \mathrm{x} \mathrm{4/28} \mathrm{days).} \mathrm{To} \mathrm{prevent}$ arachnoiditis, intrathecal DX (4 mg) was administered prior to injection of LC, and oral DX ( $4 \mathrm{mg} / 12$ hours) on days 2 and 3 after LC doses. After 2 months into maintenance therapy with thalidomide, subcutaneous plasmocytomas reappeared, and a diagnosis of early second relapse was made. She was started on a rescue chemotherapy with VLD-PACE at standard doses, and also received 2 doses of intrathecal LC (50 mg x 2/14 days). Evolution was further complicated by an acute cauda equine syndrome. Cytology and FC study of the CSF ruled out leptomeningeal dissemination, serology and cultures were also negative, and no abnormal data were detected in the brain MRI scan. A diagnosis of LC toxicity was then postulated, and FC findings helped to support it.

\section{Case 2}

A 63-year-old man was diagnosed with a mixed phenotype B/myeloid acute leukemia following the WHO 2008 diagnostic criteria. He was started on an induction chemotherapy following PETHEMA protocol 2003 for high-risk ALL which includes triple intrathecal (MTX $12 \mathrm{mg}$, cytarabine $30 \mathrm{mg}$ and hydrocortisone $20 \mathrm{mg}$ ) therapy for CNS prophylaxis (days 1,22). BM remission was reached after the 3 rd consolidation therapy (minimal residual disease $<0.01 \%$ ) with the correspondent intrathecal dosages. Four months after ALL diagnosis, before consolidation therapy, the patient developed complex partial seizures with occasional generalization. A complete neurologic evaluation, including lumbar puncture and CNS TC scan, was negative, but MRI revealed a frontal and parietal enhancement suggestive of leptomeningeal disease. He was treated with methotrexate $\left(8.4 \mathrm{~g} / \mathrm{m}^{2}\right)$, thiotepa $\left(35 \mathrm{mg} / \mathrm{m}^{2}\right)$, vincristine $\left(1.4 \mathrm{mg} / \mathrm{m}^{2}\right)$ and intrathecal LC (50 mg x 4/14 days, and $50 \mathrm{mg} \times 2 / 21$ days thereafter). After finishing, he developed mild ataxia followed by subsequently slow-onset urinary and fecal incontinence and 
perineal numbness. Neurological evaluation was classified as sacral radiculopathy. Serology and cultures were negative, CSF cytology and FC ruled out leukemic infiltration and, contrast-enhanced MRI scan of the brain and spine confirmed these data, and also discarded arachnoiditis. A diagnosis of LC toxicity was then postulated.

Table 1 summarizes the patients' clinical symptoms and CSF data obtained after the administration of the 7th, 8th and 10th LC doses (Patient 1), and 5th, 6th and 7th LC doses (Patient 2).

\section{Results}

In order to monitor leptomeningeal disease, the 2 patients had serial evaluations of the CSF during the course of the disease. CSF samples were obtained before every intrathecal administration of LC, and were manipulated as previously described [3]. One hundred microliters of the sample were used for cell-count, using the DNA dye marker DRAQ5 and fluorospheres with a previously known concentration. The remaining CSF volume was used for cell immunophenotyping and identification of myeloma cells (CD38/CD56/CD19/ CD45) in patient 1, and leukemic cells (CD5/CD34/CD45/ CD7) in patient 2. A FACSCalibur flow cytometer (Becton Dickinson, San Jose, CA) equipped with the CellQuest software program was used to run samples. Acquisition was stopped when the entire sample volume was acquired. No gating strategy was performed. Files were analyzed using the INFINICYT software program (Cytognos).

After initiation of intrathecal therapy, all CSF samples had $<2$ cells $/ \mathrm{mm}^{3}$. The immunophenotype identified T lymphocytes, without data of CNS involvement by either acute leukemia or multiple myeloma. Besides, in both patients, after the 10th and 6th doses of LC (case 1 and 2, respectively), an increasing number of events crossing the forward/ side scatter dot-plot became clear. These events were located above the cells region, and had a very heterogeneous size and granularity that ranged from very small/ $\mathrm{SSC}^{\text {low }}$ particles to large/SSC ${ }^{\text {hi }}$ particles (Fig. 1). DRAQ5 staining allowed an easy discrimination from the CSF cell compartment. The suspicion of depot particles was confirmed with the cytological examination of the CSF on a cytospin.

In an attempt to establish a relationship between the depo-foam particles found in the CSF and toxicity, we performed a flow cytometry quantitative approach. For each patient, an enumeration of the events corresponding to cells and depot particles was performed but, in order to exclude debris, we only considered events with the same or bigger size than lymphocytes. Following the same criterion, we also made a retrospective analysis of a set of stored datafiles from CSF samples of 2 groups of patients. As negative controls, $15 \mathrm{CSF}$ samples from patients diagnosed of several hematological malignancies who had not received LC.
Seven CSF from patients diagnosed of solid tumors with leptomeningeal carcinomatosis who had received among 1 and 4 doses of LC served as positive controls. In all of them, the flow cytometry acquisition protocol was the same than that used for the 2 patients of the study: 100 microliters of sample, and acquisition of the entire sample volume.

Analyses of the data showed a dramatic increase in the number of depo-foam events between the 8th and 10th LC doses in Patient 1 (774 to 13,888), and the 7th and 8th LC doses in Patient 2 (1,013 to 6,890). The median number of events within the depo-foam region was 10 (range 0 - 14) in the negative control, and 1,738 (range $294-5,735$ ) in the positive control group.

\section{Discussion}

LC is a formulation of cytarabine that allows a slow and prolonged liberation of this drug, maintaining cytotoxic levels in the CSF for a longer period of time than the standard formulation [8]. It is being used for treatment and prophylaxis of CNS involvement in many hematological malignancies, mainly ALL and lymphomas [9, 10], but also as therapy for solid tumor neoplastic meningitis [11]. Side effects with LC are not common $[12,13]$, but moderate to severe neurotoxicity has been reported [14-17]. Central (headache, vertigo, seizures, intracranial hypertension, etc...) or peripheral (cauda equina syndrome, radiculopathy) neurological symptoms may develop, but they are not specific and infections or cerebrovascular and metabolic diseases may also be responsible. Most of them have specific therapy choices, but leptomeningeal relapse and toxicity secondary to LC lead to dramatically opposite therapeutic decisions.

As seen in our patients, flow cytometry evaluation of the CSF was very useful to identify the accumulation of LC depot particles. Several reports had previously discussed the use of FC for making an early detection of CNS involvement, even before the onset of clinical symptoms [18]. In patients with high-grade B-cell lymphoma [4-7], the FC study of the CSF showed a better sensitivity and specificity than classical cytological examination. According to our data, conventional FC evaluation of the CSF can also be useful to identify accumulation of depot formulation of LC, as detected on the same FSC/SSC dot-plot used for the identification of CSF cell populations. There is no need to increase the CSF volume obtained for FC studies, and the only preventive measure is to avoid cell gating in the acquisition process. From a technical point of view it should be noted that, although cells are well-characterised through DNA staining using DRAQ5 dye, no specific marker has been used to identify depot liposomes. Their wide range of size ( 3 to $30 \mu \mathrm{m}$ ) locates them in a very heterogeneous distribution in the FSC/SSC dot-plot and it may be very difficult to distinguish the smallest depot particles from erythrocytes 
or debris. However, exclusion of the small events, and use of the same region for analysis of serial CSF samples, may be very useful for making a comparative quantification of the number of liposomes in the CSF. In contrast to cytology examination of the CSF, FC would be able to detect unexpected increases in their concentration that could eventually lead to neurotoxicity. Detection of liposomes in the CSF of patients who have received several doses of LC is not synonymous of toxicity since, as seen in the cases from the positive control group, most of them showed a higher number of depo-foam events than that in our patients. However, an abrupt onset of neurological clinical symptoms and a dramatic increase in the number of $\mathrm{FC}$ events associated to drug particles may lead to diagnosis of toxicity.

Some clinical procedures have been addressed to reduce the impact of chemical meningitis due to LC toxicity, such as the administration of dexamethasone one day before and 4 days after LC $[9,10]$. Besides, a CSF flow study prior to the intrathecal administration of the drug would help to identify areas of abnormal CSF flow [19] that may prevent a homogeneous distribution of the agent, potentially decreasing efficacy and increasing toxicity. Focal irradiation of sites of CSF flow obstruction would avoid such problems, but, unfortunately, CSF flow study is not widely available [20].

In the meantime, it would be desirable to establish which amount of liposomes should not be exceeded in the CSF for not-developing neurological toxicity. This is particularly important since early interruption of drug administration seems to be crucial for recovering from the neurological deficits and preventing chronic disablements.

In summary, we believe that serial quantitative FC studies of the CSF of patients receiving this drug formulation, would allow preventing accumulation of liposomes that could lead to toxicity. Further studies with a large number of patients are necessary to confirm our FC findings, but clinicians could use FC information as a guide to increase the interval between doses or interrupt treatment definitively.

\section{Conflicts of Interest}

Nothing to report.

\section{References}

1. Recht L, Mrugala M. Neurologic complications of hematologic neoplasms. Neurol Clin. 2003;21(1):87-105.

2. Glass J. Neurologic complications of lymphoma and leukemia. Semin Oncol. 2006;33(3):342-347.

3. Kraan J, Gratama JW, Haioun C, Orfao A, Plonquet A, Porwit A, Quijano S, et al. Flow cytometric immunophenotyping of cerebrospinal fluid. Curr Protoc Cytom.
2008; Chapter 6:Unit 625.

4. Hegde U, Filie A, Little RF, Janik JE, Grant N, Steinberg SM, Dunleavy K, et al. High incidence of occult leptomeningeal disease detected by flow cytometry in newly diagnosed aggressive B-cell lymphomas at risk for central nervous system involvement: the role of flow cytometry versus cytology. Blood. 2005;105(2):496-502.

5. Schroers R, Baraniskin A, Heute C, Vorgerd M, Brunn A, Kuhnhenn J, Kowoll A, et al. Diagnosis of leptomeningeal disease in diffuse large B-cell lymphomas of the central nervous system by flow cytometry and cytopathology. Eur J Haematol. 2010;85(6):520-528.

6. Alvarez R, Dupuis J, Plonquet A, Christov C, CopieBergman C, Hemery F, Gaillard I, et al. Clinical relevance of flow cytometric immunophenotyping of the cerebrospinal fluid in patients with diffuse large B-cell lymphoma. Ann Oncol. 2011 Sep 30. doi: 10.1093/annonc/mdr436.

7. Bromberg JE, Breems DA, Kraan J, Bikker G, van der Holt B, Smitt PS, van den Bent MJ, et al. CSF flow cytometry greatly improves diagnostic accuracy in CNS hematologic malignancies. Neurology. 2007;68(20):16741679.

8. Glantz MJ, LaFollette S, Jaeckle KA, Shapiro W, Swinnen L, Rozental JR, Phuphanich S, et al. Randomized trial of a slow-release versus a standard formulation of cytarabine for the intrathecal treatment of lymphomatous meningitis. J Clin Oncol. 1999;17(10):31103116.

9. Jabbour E, O'Brien S, Kantarjian H, Garcia-Manero G, Ferrajoli A, Ravandi F, Cabanillas M, et al. Neurologic complications associated with intrathecal liposomal cytarabine given prophylactically in combination with high-dose methotrexate and cytarabine to patients with acute lymphocytic leukemia. Blood. 2007;109(8):32143218.

10. Gokbuget N, Hartog CM, Bassan R, Derigs HG, Dombret H, Greil R, Hernandez-Rivas JM, et al. Liposomal cytarabine is effective and tolerable in the treatment of central nervous system relapse of acute lymphoblastic leukemia and very aggressive lymphoma. Haematologica. 2011;96(2):238-244.

11. Jaeckle KA, Batchelor T, O’Day SJ, Phuphanich S, New P, Lesser G, Cohn A, et al. An open label trial of sustained-release cytarabine (DepoCyt) for the intrathecal treatment of solid tumor neoplastic meningitis. J Neurooncol. 2002;57(3):231-239. 12. Garcia-Marco JA, Panizo C, Garcia ES, Deben G, Alvarez-Larran A, Barca EG, Sancho JM, et al. Efficacy and safety of liposomal cytarabine in lymphoma patients with central nervous system involvement from lymphoma. Cancer. 2009;115(9):1892-1898.

13. Spina M, Chimienti E, Martellotta F, Vaccher E, Berretta $\mathrm{M}$, Zanet E, Lleshi A, et al. Phase 2 study of intrathe- 
cal, long-acting liposomal cytarabine in the prophylaxis of lymphomatous meningitis in human immunodeficiency virus-related non-Hodgkin lymphoma. Cancer. 2010;116(6):1495-1501.

14. Bhojwani D, Pui CH. Intrathecal liposomal cytarabine: more friend than foe? Leuk Lymphoma. 2008;49(8):1427-1430.

15. Gallego Perez-Larraya J, Palma JA, Carmona-Iragui M, Fernandez-Torron R, Irimia P, Rodriguez-Otero P, Panizo C, et al. Neurologic complications of intrathecal liposomal cytarabine administered prophylactically to patients with non-Hodgkin lymphoma. J Neurooncol. 2011;103(3):603-609.

16. Hilgendorf I, Wolff D, Junghanss C, Kahl C, Leithaeuser M, Steiner B, Casper J, et al. Neurological complications after intrathecal liposomal cytarabine application in patients after allogeneic haematopoietic stem cell transplantation. Ann Hematol. 2008;87(12):1009-1012.

17. Ostermann K, Pels H, Kowoll A, Kuhnhenn J, Schlegel
U. Neurologic complications after intrathecal liposomal cytarabine in combination with systemic polychemotherapy in primary CNS lymphoma. J Neurooncol. 2011;103(3):635-640.

18. Ahluwalia MS, Wallace PK, Peereboom DM. Flow cytometry as a diagnostic tool in lymphomatous or leukemic meningitis: Ready for prime time? Cancer. 2012;118(7):1747-1753.

19. Glantz MJ, Hall WA, Cole BF, Chozick BS, Shannon CM, Wahlberg L, Akerley W, et al. Diagnosis, management, and survival of patients with leptomeningeal cancer based on cerebrospinal fluid-flow status. Cancer. 1995;75(12):2919-2931.

20. Raksin PB, Alperin N, Sivaramakrishnan A, Surapaneni $\mathrm{S}$, Lichtor T. Noninvasive intracranial compliance and pressure based on dynamic magnetic resonance imaging of blood flow and cerebrospinal fluid flow: review of principles, implementation, and other noninvasive approaches. Neurosurg Focus. 2003;14(4):e4. 